\title{
EXPANSION AND DIMENSION
}

\author{
VINCENT J. MANCUSO
}

(Received 21 May 1975)

\begin{abstract}
If $(X, \tau)$ is a topological space, $\mathscr{A}$ is a family of subsets of $X$, then the expansion of $\tau$ by $\mathscr{A}$ is the topology $\tau(\mathscr{A})$ on $X$ having $\tau \cup \mathscr{A}$ as a subbase. If $(X, \tau)$ has ptoperty $\mathscr{P}$, when does $(X, \tau(\mathscr{A}))$ have property $\mathscr{P}$ ? In this paper we answer this question for the property $\mathscr{P}$ of dimension. We apply our techniques to first countability and related properties.
\end{abstract}

\section{Introduction}

If $(X, \tau)$ is a space ${ }^{(1)}$ and $\mathscr{A}$ is a family of subsets of $X$, the expansion of $\tau$ by $\mathscr{A}$ is the topology $\tau(\mathscr{A})$ on $X$ having $\tau \cup \mathscr{A}$ as a subbase, Anderson (1965). An expansion is called finite, countable, point-finite, etc., provided the family $\mathscr{A}$ has the corresponding property. If $\mathscr{A}=\{A\}$, a singleton, then $\tau(\mathscr{A})$ is just written $\tau(A)$ and is called a simple expansion of $\tau$. Note that a member of $\tau(A)$ can be expressed as $U \cup(V \cap A)$ where $U$ and $V \in \tau$. The study of simple expansions was initiated by Levine (1964) where they are called simple extensions. Levine's paper is concerned with the following basic question. If $(X, \tau)$ has property $\mathscr{P}$, when does $(X, \tau(A))$ have $\mathscr{P}$ ? A more comprehensive study of this question was subsequently made by Borges (1967). Other authors have singled out the property $\mathscr{P}$ of connectness in their attempts to discover whether maximal connected spaces exist [Guthrie, Reynolds and Stone (1973) and Guthrie and Stone (1973)].

In this paper, we study the above question of Levine for the important property of dimension, but we do not limit ourselves to simple expansions. Finally, we will apply our techniques to first countability and related properties. The novelty of our approach is in our use of some recent results of Guthrie and Stone (1972) concerning the expansion of a mapping. ${ }^{(2)}$ More precisely, if $f$ :

(1) All spaces $(X$, ) are assumed to be Hausdorff.

( ) All mappings are continuous surjections. 
$(X, \tau) \rightarrow(Y, \sigma)$ is a mapping and $\sigma(\mathscr{A})$ is an expansion of $\sigma$ by $\mathscr{A}$, we let $f^{-1}(\mathscr{A})=\left\{f^{-1}(A) ; A \in \mathscr{A}\right\}$. If we now expand $\tau$ by $f^{-1}(\mathscr{A})$, then $f$ : $\left(X, \tau\left(f^{-1}(\mathscr{A})\right)\right) \rightarrow(Y, \sigma(\mathscr{A}))$ is still a mapping and is called the expansion of $f$ by $\mathscr{A}$ or simply the expansion of $f$ [Guthrie and Stone (1972)].

\section{Expansion and dimension}

Borges (1967) defines the notion of an infinite expansion of a space $(X, \tau)$ as follows. If $\mathscr{A}=\left\{A_{\alpha}\right\}$ is a family of subsets of $X$ and $\mathscr{F}=\left\{\tau\left(A_{\alpha}\right)\right\}$ is a corresponding family of simple expansions of $\tau$, then the topology on $X$ generated by $\mathscr{F}$ is called the $\mathscr{F}$-extension of $\tau$. This topology is denoted by $\Lambda$. As the following proposition shows, the topology $\wedge$ is the same as the expansion of $\tau$ by $\mathscr{A}$.

Proposition 2.1. Let $(X, \tau)$ be a space, $\mathscr{A}=\left\{A_{\alpha} ; \alpha \in \Gamma\right\}$ a family of subsets of $X$ and $\mathscr{F}=\left\{\tau\left(A_{\alpha}\right)\right\}$ a corresponding family of simple expansions of $\tau$. Then $\wedge=\tau(\mathscr{A})$.

Proof. Let $O \in \tau(\mathscr{A})$. By a lemma of Reynolds (preprint), $O=$ $\cup\left\{O^{s} \cap\left(\cap A_{\alpha}\right) ; \alpha \in s\right.$, a finite subset of $\Gamma$ and $\left.O^{s} \in \tau\right\}$. Since each $O^{s} \cap A_{\alpha} \in$ $\tau\left(A_{\alpha}\right)$, it is easy to see that $O \in \wedge$. This shows that $\tau(\mathscr{A}) \subseteq \wedge$. It is a simple exercise to establish the reverse inclusion.

We will make frequent use of the following result of Borges (1967).

Proposition 2.2. Let $(X, \tau)$ be a regular space and $A \subseteq X$. Then $(X, \tau(A))$ is regular if and only if $\operatorname{cl}(A)-A$ is closed in $(X, \tau)$.

Here and throughout this paper $\mathrm{cl}$ means closure. The reader should consult the texts of Nagata (1965) and Nagami (1970) for any dimension theory concepts not defined here. We will denote covering dimension, large inductive dimension, and small inductive dimension of a space $(X, \tau)$ by $\operatorname{dim}(X, \tau)$, Ind $(X, \tau)$ and ind $(X, \tau)$ respectively.

REMARK 2.3. It is well known that dim and Ind coincide for metric spaces [Nagata (1965), p. 27] while all three dimension concepts coincide for separable metric spaces. [Nagata (1965), p. 90].

A normal space $(X, \tau)$ is called totally normal [Dowker (1952)], if every $U \in \tau$ can be written as a union, locally finite in $U$, of open $F_{\sigma}$ sets of $(X, \tau)$. Totally normal spaces are hereditarily normal [Dowker (1952), 4.6].

THeOREM 2.4. Let $(X, \tau)$ be a totally normal space and $A \subseteq X$. If Ind $(X, \tau) \leqq n$ and $\operatorname{cl}(A)-A$ is closed in $(X, \tau)$, then $\operatorname{Ind}(X, \tau(A)) \leqq n$. 
Proof. By Proposition 2.2 and Borges (1967), Theorem $3.5(X, \tau(A))$ is hereditarily normal. Note that $X-A$ is closed in $(X, \tau(A))$ and so by Dowker (1952), p. 269, $\operatorname{Ind}(X, \tau(A)) \leqq \max \{\operatorname{Ind}(A, \tau(A) \cap A), \quad \operatorname{Ind}(X-A, \tau(A) \cap$ $(X-A))\}$. By Levine (1964), Lemma 3, $\tau(A)$ coincides with $\tau$ on both $A$ and $X-A$. Therefore, since $\operatorname{Ind}(X, \tau) \leqq n$ and Ind is monotone in totally normal spaces [Dowker (1952), Theorem 2] we have that $\operatorname{Ind}(X, \tau(A)) \leqq n$. This completes the proof.

THeOREM 2.5. Let $(X, \tau)$ be a totally normal space and $A \subseteq X$. If $\operatorname{dim}(X, \tau) \leqq n$ and $\operatorname{cl}(A)-A$ is closed in $(X, \dot{\tau})$, then $\operatorname{dim}(X, \tau(A)) \leqq n$.

Proof. The proof is the same as that of Theorem 2.4 except that we make use of Dowker (1955), 2.3 and 2.8.

The following example shows that we cannot relax the hypothesis that $\operatorname{cl}(A)-A$ be closed in $(X, \tau)$ in Theorems 2.4 and 2.5 even if $(X, \tau)$ is hereditarily Lindelöf.

EXAmple 2.6. Let $(X, \tau)$ be the real line with the right half-open interval topology and let $A$ be the set of rationals. By Proposition 2.2 and Borges (1967), Theorem $3.4(X, \tau(A))$ is not normal. Therefore, Ind $(X, \tau(A))>0$ and so $\operatorname{dim}(X, \tau(A))>0$. But $\operatorname{Ind}(X, \tau)=\operatorname{dim}(X, \tau)=0$.

The next example shows that we cannot relax the hypothesis that $(X, \tau)$ be totally normal in Theorems 2.4 and 2.5 even if $(X, \tau)$ is compact and $A$ is a point.

ExAmple 2.7. Let $(X, \tau)=[0, \omega] \times[0, \Omega]$, the Tychonoff plank and let $A=\{(\omega, \Omega)\}$. Since $X-A$ is not normal, $(X, \tau(A))$ is not normal [Borges (1967), Theorem 3.4]. Therefore, Ind $(X, \tau(A))>0$ and so $\operatorname{dim}(X, \tau(A))>0$. But Ind $(X, \tau)=\operatorname{dim}(X, \tau)=0$.

In the following theorem we will use the notation in Borges (1967), Lemma 5.5. That is, if $\left\{A_{j}\right\}$ is a countable family of subsets of $X$; let $\Lambda_{1}=\tau\left(A_{1}\right)$ and $\Lambda_{n}=\Lambda_{n-1}\left(A_{n}\right)$ for each $n$.

Theorem 2.8. Let $(X, \tau)$ be metric, $A_{j} \subseteq X$ and $\mathscr{F}=\left\{\tau\left(A_{j}\right)\right\}$ a countable family of simple expansions of $\tau$ such that $\mathrm{cl}\left(A_{j}\right)-A_{j}$ is closed in $(X, \tau)$ for each $j$. If Ind $(X, \tau) \leqq 0$, then Ind $(X, \wedge) \leqq 0$.

Proof. We first show that $\operatorname{Ind}\left(X, \wedge_{n}\right) \leqq 0$ for each $n$ and we do this by induction on $n$. The case $n=1$ is handled by Theorem 2.4 since a metric space is totally normal. Now suppose that $\operatorname{Ind}\left(X, \wedge_{n-1}\right) \leqq 0$. By Borges (1967), Lemma $5.5(\mathrm{~b})\left(X, \wedge_{n}\right)$ is regular for each $n$. Since $\Lambda_{n}=\Lambda_{n-1}\left(A_{n}\right)$ we have by Proposition 2.2 that $\mathrm{cl}_{n-1}\left(A_{n}\right)-A_{n}$ is closed in $\left(X, \wedge_{n-1}\right)$. Here, $\mathrm{cl}_{n-1}$ denotes the closure in $\wedge_{n-1}$. By Borges (1967), Lemma 5.5(d), $\left(X, \wedge_{n-1}\right)$ is metric and so Ind $\left(X, \wedge_{n}\right) \leqq 0$ by Theorem 2.4 again. 
Now let $\mathscr{B}_{n}$ be a $\sigma$-locally finite base for $\left(X, \wedge_{n}\right)$ consisting of open and closed sets. By Borges (1967), Lemma 5.5(c) and Theorem 5.2, $\mathscr{B}=\cup \mathscr{B}_{n}$ is a $\sigma$-locally finite base for $(X, \wedge)$ consisting of open and closed sets and $(X, \wedge)$ is metric; therefore, $\operatorname{Ind}(X, \wedge) \leqq 0$. This completes the. proof.

Our main theorem uses a mapping characterization of dimension originally due to Morita, but may be found embodied in Nagami (1970), Theorem 12.6.

THEOREM 2.9. (Morita). $(X, \tau)$ is metric with $\operatorname{Ind}(X, \tau) \leqq n$ if and only if there exist a metric space $(Y, \sigma)$ with $\operatorname{Ind}(Y, \sigma) \leqq 0$ and a closed mapping $f$ : $(Y, \sigma) \rightarrow(X, \tau)$ with order $\leqq n+1$ (here order of $f$ is the maximum of the cardinals of $\left.f^{-1}(x), x \in X\right)$.

THEOREM 2.10. Let $(X, \tau)$ be metric and $\mathscr{A}=\left\{A_{i}\right\}$ a countable family of subsets of $X$ such that $\mathrm{cl}\left(A_{j}\right)-A_{j}$ is closed in $(X, \tau)$ for each $j$. If Ind $(X, \tau) \leqq k$, then $\operatorname{Ind}(X, \tau(\mathscr{A})) \leqq k$.

Proof. By Theorem 2.9, there exist a metric space $(Y, \sigma)$ with Ind $(Y, \sigma) \leqq 0$ and a closed mapping $f:(Y, \sigma) \rightarrow(X, \tau)$ with order $f \leqq k+1$. Let $f:\left(Y, \sigma\left(f^{-1}(\mathscr{A})\right)\right) \rightarrow(X, \tau(\mathscr{A}))$ be the expansion of $f$ by $\mathscr{A}$. By Guthrie and Stone (1972), Theorem 7, this expansion is a closed mapping, and since it is the same function $f$, the order is unchanged. Now since $\operatorname{cl}\left(A_{j}\right)-A_{j}$ is closed in $(X, \tau)$ for each $j$, it is easy to see that $\operatorname{cl}\left(f^{-1}\left(A_{j}\right)\right)-f^{-1}\left(A_{j}\right)$ is closed in $(Y, \sigma)$ for each $j$. By Proposition 2.1, $\sigma\left(f^{-1}(\mathscr{A})\right)$ is the $\mathscr{F}$-expansion of $\sigma$ where $\mathscr{F}=\left\{\sigma\left(f^{-1}\left(A_{i}\right)\right)\right\}$. Therefore, by Theorem 2.8 , Ind $\left(Y, \sigma\left(f^{-1}(\mathscr{A})\right)\right) \leqq 0$. Then by [Borges (1967), Theorem 5.2] $\left(Y, \sigma\left(f^{-1}(\mathscr{A})\right)\right.$ is metric and so Ind $(X, \tau(\mathscr{A})) \leqq k$ by applying Theorem 2.9 again. This completes the proof.

REMARK 2.11. Theorem 2.10 holds if Ind is everywhere replaced by dim. This follows from Remark 2.3 and Borges (1967), Theorem 5.2.

A metric space $(X, \tau)$ is countable dimensional if $(X, \tau)$ is a countable union of subspaces $X_{i}$ such that Ind $X_{i} \leqq 0$ for each $i$ [Nagata (1965), p. 162].

Theorem 2.12. [Nagata (1965), p. 169]. A space $(X, \tau)$ is metric and countable dimensional if and only if there exist a metric space $(Y, \sigma)$ with Ind $(Y, \sigma) \leqq 0$ and a closed mapping $f:(Y, \sigma) \rightarrow(X, \tau)$ such that $f^{-1}(x)$ is finite for each $x \in X$.

THEOREM 2.13. Let $(X, \tau)$ be metric and $\mathscr{A}=\left\{A_{i}\right\}$ a countable family of subsets of $X$ such that $\operatorname{cl}\left(A_{j}\right)-A_{j}$ is closed in $(X, \tau)$ for each $j$. If $(X, \tau)$ is countable dimensional, so is $(X, \tau(\mathscr{A}))$.

Proof. The same as the proof of Theorem 2.10 except that we make use of Theorem 2.12 instead of Theorem 2.9. 
The next example has been gleaned from an example in Borges (1967) and shows that the countability of the family $\mathscr{A}$ in Theorem 2.10 is essential.

EXAmple 2.14 Let $X$ be those points in the plane both of whose coordinates are irrational and let $\tau$ be the usual topology of the plane relative to $X$. Let $\sigma$ be Sorgenfrey's half-open rectangle topology of the plane relative to $X$. For each $(a, b) \in X$, let $A_{a b}=\{(x, y) \in X ; a \leqq x<a+1, b \leqq x<b+1\}$. It is easy to see that $(X, \sigma)=(X, \tau(\mathscr{A}))$ where $\mathscr{A}=\left\{A_{a b}\right\}$. Note that $\mathrm{cl}\left(A_{a b}\right)-A_{a b}$ is closed in $(X, \tau)$ for each $(a, b) \in X$. However, $(X, \sigma)$ is not normal and so Ind $(X, \sigma)$ and $\operatorname{dim}(X, \sigma)>0$. But $\operatorname{Ind}(X, \tau)=\operatorname{dim}(X, \tau)=0$.

Remark 2.15. Dowker (1955) defines the local dimension of a space $(X, \tau)$. It is an easy consequence of 3.3 and 3.4 of that paper that if $(X, \tau)$ is hereditarily paracompact, we may replace Ind by loc Ind and dim by loc dim in Theorems 2.4 and 2.5 respectively.

The next example is mentioned as an afterthought in case the reader might wonder if the simple expansion of a space can have dimension strictly less than the given space. Unfortunately, our space $(X, \tau)$ isn't even normal.

Example 2.16. Let $(X, \tau)$ be the Dieudonné plank as described in Steen and Seebach (1970), p. 108. Let $A$ be the union of the top and right side of the plank. Since $A$ is closed in $(X, \tau),(X, \tau(A))$ is the disjoint union of $A$ and $X-A$ which are each open and closed in $(X, \tau(A))$. Since $A$ and $X-A$ are discrete subspaces of $(X, \tau)$, it is easy to see that $\ln A=\operatorname{Ind}(X-A)=0$. Now $\tau$ and $\tau(A)$ coincide on both $A$ and $X-A$. Therefore, we have that $\operatorname{Ind}(X, \tau(A))=0$ by Dowker (1952), 5.1. But Ind $(X, \tau)>0$ since $(X, \tau)$ is not normal.

THEOREM 2.17. Let $(X, \tau)$ be hereditarily Lindelöf, ind $(X, \tau)=0$ (resp. ind $(X, \tau)=1)$. If $A \subseteq X$ and $\mathrm{cl}(A)-A$ is closed in $(X, \tau)$, then ind $(X, \tau(A))=0$ (resp. ind $(X, \tau(A))=1)$.

Proof. $n=0$ : By Engelking (1968), Theorem 10, p. 269, ind $(X, \tau)=0$ and $\operatorname{Ind}(X, \tau)=0$ are equivalent. Now an hereditarily Lindelöf space is totally normal (Dowker (1952), 4.2]. Note that by Proposition 2.2 and Borges (1967), Theorem 3.8, it follows that $(X, \tau(A))$ is a Lindelöf space. Then ind $(X, \tau(A))=0$ by Theorem 2.4 .

$n=1$ : This case is proved similarly except that we use Engelking (1968), Theorem 5, p. 280 in place of Theorem 10, p. 269 of that paper. This completes the proof.

The following example shows that $\operatorname{cl}(A)-A$ be closed in $(X, \tau)$ is essential in Theorem 2.17. In fact, for $n=0$, this condition is easily seen to be equivalent to ind $(X, \tau(A))=0$. The reader can convince himself of this by Proposition 2.2 and the fact that ind $(X, \tau)=0$ implies $(X, \tau)$ is regular. 
Example 2.18. Let $(X, \tau)$ and $A$ be as in Example 2.6. Now ind $(X, \tau)=0$ but $(X, \tau(A))$ is not regular. Therefore, ind $(X, \tau(A))>0$.

THEOREM 2.19. Let $(X, \tau)$ be hereditarily Lindelöf, ind $(X, \tau)=0$ and $\mathscr{A}=$ $\left\{A_{\alpha} ; \alpha \in \Gamma\right\}$ a family of subsets of $X$ such that $\operatorname{cl}\left(A_{\alpha}\right)-A_{\alpha}$ is closed in $(X, \tau)$ for each $\alpha \in \Gamma$. Then ind $(X, \tau(\mathscr{A}))=0$.

Proof. By Theorem 2.17, ind $\left(X, \tau\left(A_{\alpha}\right)\right)=0$ for each $\alpha \in \Gamma$. Let $\mathscr{B}_{\alpha}$ be a clopen base of $\left(X, \tau\left(A_{\alpha}\right)\right)$ for each $\alpha \in \Gamma$ and let $\mathscr{B}=\left\{\cap\left\{B_{\alpha} ; B_{\alpha} \in \mathscr{B}_{\alpha}, \alpha \in s, s\right.\right.$ a finite subset of $\Gamma\}\}$. Let $\wedge$ be the topology on $X$ generated by $\left\{\tau\left(A_{\alpha}\right) ; \alpha \in \Gamma\right\}$. Clearly, $\mathscr{B}$ is a clopen base for $(X, \wedge)$ and hence for $(X, \tau(\mathscr{A}))$ by Proposition 2.1. Therefore, ind $(X, \tau(\mathscr{A}))=0$ and we are done.

Example 2.20. This example appears in Franklin (1969) in a different context. Let $(X, \tau)$ be $\beta N$, the Stone-Cech compactification of the natural numbers $N$. Let $N_{p}=\{p\} \cup N$ for each $p \in \beta N-N$ and let $\mathscr{A}=\left\{N_{p}\right\}$. Observe that the space $F$ described in Franklin's paper is just the expansion of $\tau$ by $\mathscr{A}$. By Nagami (1970), Theorem 10-3 and Proposition 8.4 it follows that ind $\beta N=0$. But ind $F>0$. Note that $\mathrm{cl}_{\beta N}\left(N_{p}\right)-N_{p}$ is not closed in $\beta N$, nor is $\beta N$ hereditarily normal [Orevkov (1962)].

\section{Application to first countability}

In this last section we show that the properties: first countable, point countable base and bi-sequential are preserved by countable expansions, while the property Frechet is preserved by countable point-finite expansions.

THEOREM 3.1. Let $(X, \tau)$ have any one of the following properties:

(a) First countable

(b) Point countable base

(c) Bi-sequential.

If $\mathscr{A}=\left\{A_{n}\right\}$ is a countable family of subsets of $X$, then $(X, \tau(\mathscr{A}))$ has the corresponding property.

Proof. (a) if $\left\{V_{i}\right\}$ is a countable local base at $x$ in $(X, \tau)$, it is easy to see that $\left\{V_{i}\right\} \cup\left\{V_{i} \cap A_{n}\right\}$ is a countable local base at $x$ in $\left(X, \tau\left(A_{n}\right)\right)$ and so $\left(X, \tau\left(A_{n}\right)\right)$ is first countable for each $n$. Now by Proposition 2.1 and Levine (1966), Corollary 9 we have that $(X, \tau(\mathscr{A}))$ is first countable.

(b) The proof for point countable base is very similar to the proof of (a) and is left to the reader.

(c) By a result of Michael (1972), Theorem 3.D.2, there exist a metric space (equivalently, a first countable space) $(Y, \sigma)$ and a bi-quotient mapping $f$ : 
$(Y, \sigma) \rightarrow(X, \tau)$. Let $f:\left(Y, \sigma\left(f^{-1}(\mathscr{A})\right)\right) \rightarrow(X, \tau(\mathscr{A}))$ be the expansion of $f$ by $\mathscr{A}$. By Guthrie and Stone (1972), Theorem 3, this expansion is bi-quotient and by $(\mathrm{a}),\left(Y, \sigma\left(f^{-1}(\mathscr{A})\right)\right)$ is first countable. By applying Michael's result again, we have that $(X, \tau(\mathscr{A}))$ is bi-sequential. This completes the proof.

THEOREM 3.2. Let $(X, \tau)$ be a Frechet space and $\mathscr{A}=\left\{A_{n}\right\}$ a countable family of subsets of $X$ such that $\mathscr{A}$ is point-finite in $(X, \tau)$. Then $(X, \tau(\mathscr{A}))$ is a Frechet space.

Proof. By a result of Franklin (1965), Proposition 2.4, there exist a metric space (equivalently, a first countable space) $(Y, \sigma)$ and a pseudo-open mapping $f:(Y, \sigma) \rightarrow(X, \tau)$. The proof now parallels that of Theorem 3.1 (c) except that we use Corollary 2B in Guthrie and Stone (1972) and then apply Franklin's result again.

The following example shows that the countability of $\mathscr{A}$ in the above theorems and the point-finiteness in Theorem 3.2 are essential even if $(X, \tau)$ is separable metric.

Example 3.3. Let $(X, \tau)$ be the reals with the usual topology. Let $A_{U}=$ $\{0\} \cup U$ where $U$ is a usual neighborhood of the sequence $\{1 / n\}$. Let $\mathscr{A}=\left\{A_{U}\right\}$. Then $(X, \tau(\mathscr{A}))$ is just Example 1.8 in Franklin (1965) where it is shown that $(X, \tau(\mathscr{A}))$ is not Frechet (see Franklin (1965), Example 2.2).

REMARK 3.4. Theorem 3.1 also holds for the property countable bisequential Siwiec (1971). The proof would be similar to that of Theorem 3.1 (c) except that we use Theorem 4.4 in Siwiec (1971) in place of Michael's result and Theorem 4 in place of Theorem 3 in Guthrie and Stone (1972).

\section{References}

D. R. Anderson (1965), 'On connected irresolvable Hausdorff spaces', Proc. Amer. Math. Soc. 16, 463-466.

C. J. Borges (1967), 'On extensions of topologies', Canad. J. Math. 19, 474-487.

C. H. Dowker (1952), 'Inductive dimension of completely normal spaces', Quart. J. Math. (2) 4, 267-281.

R. Engelking (1968), Outline of General Topology (Wiley, New York).

S. P. Franklin (1965), 'Spaces in which sequences suffice', Fund. Math. 57, 107-116.

S. P. Franklin (1969), 'On two questions of Moore and Mrowka', Proc. Amer. Math. Soc. 21, 597-599.

J. A. Guthrie and H. E. Stone (1972), 'Expansions of mappings', Second Pittsburgh International Conference, Lecture Notes in Mathematics, Springer-Verlag 378, 164-171.

J. A. Guthrie and H. E. Stone (1973), 'Spaces whose connected expansions preserve connected subsets', Fund. Math. 80, 91-100.

J. A. Guthrie, D. F. Reynolds and H. E. Stone (1973), 'Connected expansions of topologies', Bull. Austral. Math. Soc. 9, 259-265. 
N. Levine (1964), 'Simple extensions of topologies', Amer. Math Monthly 71, 22-25.

N. Levine (1966), 'On families of topologies for a set', Amer. Math, Monthly 73, 358-361.

E. Michael (1972), 'A quintuple quotient quest', Gen. Topology Appl. 2, 91-138.

K. Nagami (1970), Dimension Theory (Academic Press, New York).

J. Nagata (1965), Modern Dimension Theory (Wiley, New York).

Ju. Orevkov (1962), 'Totally bounded metric spaces', Soviet Math. Dokl. 3, 386-387.

D. F. Reynolds (preprint) 'Preservation of connectedness under extensions of topologies'.

F. Siwiec (1971), 'Sequence covering and countably bi-quotient mappings', Gen. Topology Appl. 1, 143-154.

L. A. Steen and J. A. Seebach, Jr. (1970), Counterexamples in Topology (Holt, Rhinehart and Winston, New York).

Department of Mathematics,

St. John's University,

Jamaica, New York, 11439,

U.S.A. 